\section{MS26-P5 When alternative layer stackings cause commensurate structures to co-exist the interpretation of the diffraction data need not be unique.}

\section{A. David Rae ${ }^{1}$}

\section{Australian National University}

email: rae@rsc.anu.edu.au

Refinements of crystal structures with commensurate stacking faults should recognize that the structure is not everywhere the same and the structure can change coherently from one structure to another across an interface between adjacent layers. Assumptions are commonly used to constrain a refinement by placing the structure in a particular category consistent with the capabilities of a particular program. However this categorization can restrict the success of a refinement. The first assumption is that the options for a layer can be constructed using a symmetry operation commensurate with the crystal lattice and either a single reference layer or a single commensurately modulated structure. The second is that a clear distinction can be made between twinning and disorder. The twinning concept can include allo twins, ie the diffraction pattern has independent intensity contributions from different selections of layers corresponding to different ordered structures. Problems associated with these constraints are minimized when a single orientation of a single component structure dominates the diffraction data. However a systematic error still remains. Pseudo equivalent reflections of a disordered structure containing the various packing options can be used to project out the contributions to the scattering density from different irreducible representations of a commensurate parent structure and hence refine a correlation coefficient (range 0 to 1 ) between the component structures. Recent structure determinations will be used to illustrate this.

Keywords: polymorphs, stacking faults, refinement

\author{
MS26-P6 Influence of the oxygen \\ concentration on crystal growth and \\ structure of the $\mathrm{BaCuSi}_{2} \mathrm{O}_{6 \pm \delta}$ and \\ $\mathrm{Ba}_{1-\mathrm{x}} \mathrm{Sr}_{\mathrm{x}} \mathrm{CuSi}_{2} \mathrm{O}_{6 \pm \delta}$ spin dimer compounds \\ Natalija van Well ${ }^{1}$, Pascal Puphal ${ }^{2}$, Björn Wehinger ${ }^{1,3}$, Mariusz \\ Kubus $^{1,4}$, Dmitry Chernyshov ${ }^{5}$, Denis Sheptyakov ${ }^{1}$, Franz Ritter ${ }^{2}$, \\ Wolf Assmus ${ }^{2}$, Cornelius Krellner ${ }^{2}$, Jürg Schefer ${ }^{1}$, Christian \\ Rüegg $g^{1,3}$
}

1. Laboratory for Neutron Scattering and Imaging, Paul Scherrer Institute, CH-5232 Villigen, Switzerland

2. Physikalisches Institut, Goethe-Universität Frankfurt, D-60438 Frankfurt am Main, Germany

3. Department for Quantum Matter Physics, University of Geneva, CH-1211 Geneva, Switzerland

4. Department of Chemistry and Biochemistry University of Bern, CH-3012 Bern, Switzerland

5. Swiss-Norwegian Beam Lines at the European Synchrotron Radiation Facility, 38042 Grenoble, France

email: Natalija.van-well@psi.ch

By using the crystal growth method with $\mathrm{O}_{2}$-partial pressure the new $\mathrm{Ba}_{1-x} \mathrm{Sr}_{x} \mathrm{CuSi}_{2} \mathrm{O}_{6 \pm \delta}$ mixed system can be prepared for $\mathrm{x} \leq 0.3$. The crystal growth of $\mathrm{BaCuSi}_{2} \mathrm{O}_{6}$ is also possible at a temperature of $1150^{\circ} \mathrm{C}$ by using an oxygen pressure of around 1 bar. The particular challenge of crystal growth of this material is that for the existing oxygen networks oxygen partial pressure operates as control parameter. $\mathrm{BaCuSi}_{2} \mathrm{O}_{6 \pm \delta}$ and $\mathrm{Ba}_{1-\mathrm{x}} \mathrm{Sr}_{\mathrm{x}} \mathrm{CuSi}_{2} \mathrm{O}_{6 \pm \delta}$ mixed crystals have the same tetragonal structure type $I 4$ lacd down to low temperature, which has only one type of dimer layers. First results from single crystal structure diffraction of the new $\mathrm{BaCuSi}_{2} \mathrm{O}_{6+8}$ show that, in contrast to $\mathrm{Sr}$ doped crystals of this mixed system, the incommensurate structure is present at low temperature (see Fig. 1). $\mathrm{BaCuSi}_{2} \mathrm{O}_{6}$ is a model material for studying Bose-Einstein condensation (BEC) of magnons in high magnetic fields. It is also a quasi-two dimensional spin dimer system. The material is observed to have a singlet ground state in zero magnetic field with a large gap to the lowest excited triplet states $[1,2]$. The quantum critical point at around $23 \mathrm{~T}$ and $\mathrm{T}=0 \mathrm{~K}$ separates the quantum paramagnetic regime from the ordered state $[3,4]$. The investigation of the proportions of oxygen in the compounds shows a variation of the imbedded oxygen content $( \pm \delta)$. A detailed understanding of the crystal structure, the incommensurately modulated structure depending on the oxygen content will enable studying the spin dynamics of field-induced order states in this model magnetic compound of high current interest with only one type of dimer layers, showing the same distance between the $\mathrm{Cu}$ atoms, in the structure.

[1] Y. Sasago, K. Uchinokura, A. Zheludev, and G. Shirane, Phys. Rev. B 55, 8357 (1997) [2] C. Rüegg, D. F. McMorrow, B. Normand, H. M. Rønnow, S. E. Sebastian, I. R. Fischer, C. D. Batista, S. N. Gvasaliya, C. Niedermayer, J. Stahn, Phys. Rev. Lett. 98, 017202 (2007) [3] M. Jaime, V. F. Correa, N. Harrison, C. D. Batista, N. Kawashima, Y. Kazuma, G. A. Jorge, R. Stern, I. Heinmaa, S. A. Zvyagin, Y. Sasago, K. Uchinokura, Phys. Rev. Lett. 93, 087203 (2004) [4] S. E. Sebastian, N. Harrison, C. D. Batista, L. Balicas, M. Jaime, P. A. Sharma, N. Kawashima, and I. R. Fisher, Nature (London) 441, 617 (2006) 